\title{
Positron annihilation studies of some odd-n fatty acids
}

\author{
W.W. WALKER
}

Department of Physics and Astronomy, The University of Alabama, Tuscaloosa, AL 35487, U.S.A.

\begin{abstract}
Positron lifetime measurements have been made as a function of temperature in solid samples of fatty acids having chain lengths of $15,17,19,21$, and 23 carbon atoms in order to complement a previous study of even-n fatty acids (1). The well-known solid-solid transitions occurring several degrees below the melting point were clearly observed in the positron data. Good agreement with published values (2) for the transition temperatures was obtained. Comparison of the lifetime data for odd- $n$ acids with that obtained for even- $n$ acids reveals a pronounced odd-even effect, especially in the dependence of the long lifetime intensity $I_{3}$ on chain length. These variations, which are similar to the melting point alternation of even-n and odd- $n$ fatty acids, are attributed to free-volume variations in this homologous series.
\end{abstract}

\section{INTRODUCTION}

Fatty acids always show polymorphism, with $\alpha$ and $B$ being the most commonly encountered forms (2). The $\alpha$ form has $x$-ray diffraction long-spacings which are referred to as $C$-spacings for even $n$ and $C$ '-spacings for odd $n$, while the $B$ form has B-spacings for even $n$ and $B$ 'spacings for odd $n$. Of importance in this study is the fact that each of the samples of odd-n fatty acids studied initially had B' spacings and each underwent a solid-solid transition from the $\mathrm{B}^{\prime}$ to the $\mathrm{C}^{\prime}$ form a few degrees below the melting point.

\section{EXPERIMENTAL}

Samples of fatty acids were obtained from Sigma Chemical Company and had a stated purity of $99 \%$. Positron lifetimes were measured with an apparatus which has been described previously (3). The program Positronfit Extended (4) was used to analyze the data into three components. Differential scanning calorimetry was performed using a Perkin-Elmer DSC-1B. $\mathrm{X}$-ray patterns were obtained using a Philips XRG3100 x-ray diffractometer.

\section{RESULTS}

The results of the positron lifetime measurements as a function of temperature are shown in Figures 1-3. The solid lines are drawn to guide the eye. The most noteworthy features in the data are the abrupt changes in the long lifetime $\tau_{3}$ and its intensity $I_{3}$ which occur in each sample several degrees below the melting point. 


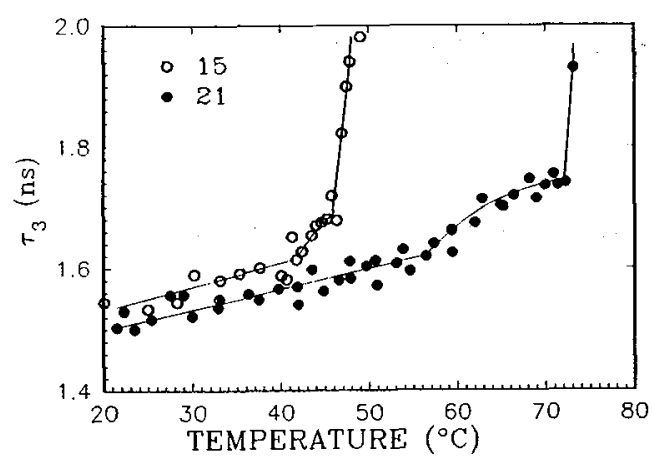

Figure 1. Dependence of positron lifetime on temperature for acids with $n=15$ and 21 .

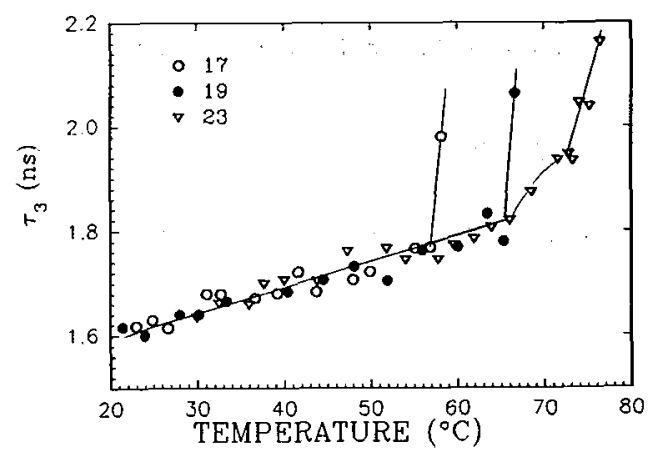

Figure 2. Dependence of positron lifetime on temperature for acids with $n=17,19,23$.

\section{DISCUSSION}

Table 1 gives a comparison of the transition temperatures obtained by positron measurements with previously published values. (2). Agreement is best for the $\tau_{3}$ data (within $1.0^{\circ}$ ). It is not ciear why these transitions are observed at slightly lower temperatures in the $I_{3}$ data than in the $\tau_{3}$ data. In addition to these relatively sharp $B^{\prime}$ to $C^{\prime}$ transitions, the $\tau_{3}$ data for $n=15$, $r=21$, and $n=23$ and the $I_{3}$ data for $n=15, n=17$, and $n=19$ exhibit "shoulders" at temperatures just below the B' to C' transition. These can possibly be attributed to "premelting" effects which have been observed in the fatty acids (5).

Table 1. Temperatures for B' to C'Transition

\begin{tabular}{|c|c|c|c|}
\hline No. of Carbons & Reference 2 & From $\tau_{3}$ Data & From $\mathrm{I}_{3}$ Data \\
\hline 15 & $46^{\circ} \mathrm{C}$ & $46^{\circ} \mathrm{C}$ & $44.5^{\circ} \mathrm{C}$ \\
\hline 17 & $56^{\circ} \mathrm{C}$ & $57{ }^{\circ} \mathrm{C}$ & $55.5^{\circ} \mathrm{C}$ \\
\hline 19 & $66^{\circ} \mathrm{C}$ & $66^{\circ} \mathrm{C}$ & $64.5^{\circ} \mathrm{C}$ \\
\hline 21 & $72^{\circ} \mathrm{C}$ & $72.5^{\circ} \mathrm{C}$ & $71.5^{\circ} \mathrm{C}$ \\
\hline 23 & $77.5^{\circ} \mathrm{C}$ & $76.5^{\circ} \mathrm{C}$ & $76.5^{\circ} \mathrm{C}$ \\
\hline
\end{tabular}

Shown in Figure 4 are the $\mathrm{I}_{3}$ data for samples measured at $20^{\circ} \mathrm{C}$ and also the melting point for these same samples. Both $I_{3}$ and the melting point exhibit alternating values with the odd $\mathbf{n}$ acids having the higher $I_{3}$ values and the lower melting points. The alternating $I_{3}$ values show a downward trend with increasing $n$ while the alternating melting points show an upward trend. It shouid be emphasized that all odd-n samples are in the B' form whereas the even-n samples shown for comparison [1] are in the B form. (The absence of data for $n=16$ is. due to the inability to produce a sample in the form having B spacings.) Although not shown, $\tau_{3}$ exhibits a less pronounced odd-even effect than does $\mathrm{I}_{3}$, with $\tau_{3}$ alternating about a constant value of about $1.5 \mathrm{~ns}$. In general, the odd-n acids have the higher $\tau_{3}$ values. 


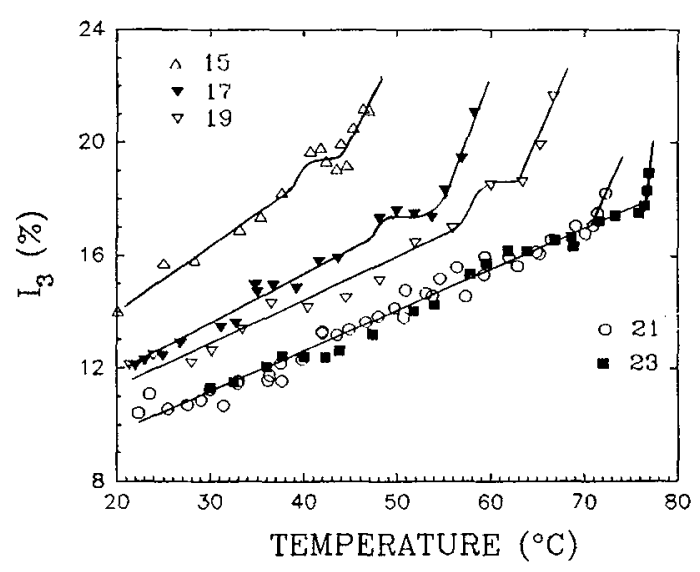

Figure 3. Dependence of positron lifetime intensity $I_{3}$ on temperature.

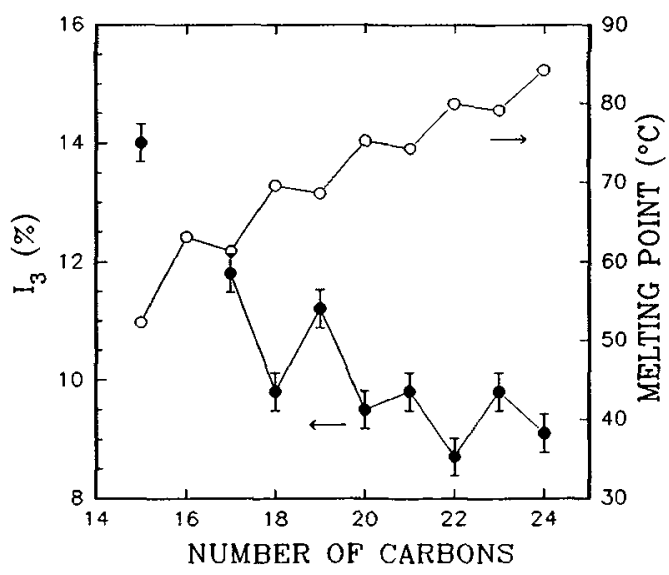

Figure 4. Comparison of the dependence of $\mathrm{I}_{3}$ and melting point on the number of carbons.

It is generally accepted (2) that alternation of properties between even-n and odd-n members of many homologous series of long-chain compounds can be accounted for by the angle of tilt of the long chain. Such tilting gives rise to a significant geometric difference between the crystal structures, resulting in the zigzag behavior observed when the melting point is plotted as a function of chain length. In summary, the general trends for increasing chain length are: the melting point tends to increase while $I_{3}$ tends to decrease, and $\tau_{3}$ alternates about an approximately constant value. Since higher melting point implies more tightly bound molecules, it would be expected that free volume available to form positronium would generally decrease with increasing chain length. It is known (6) that molecules of evennumbered fatty acid are packed more closely in the crystal lattice than those of the next higher odd-numbered acid. This is in agreement with the positron results, especially the $I_{3}$ data.

\section{CONCLUSIONS}

The well-known B' to C' transitions occurring several degrees below the melting point of each acid were clearly observed in the positron data for each of the samples at temperatures in good agreement with those observed by other techniques. Comparison of the lifetime data for odd-n acids with that obtained for the even-n acids reveals an odd-even effect in the dependence of the long lifetime intensity $I_{3}$ on chain length, similar to the melting point alternation of even-n and odd- $n$ fatty acids. These variations are attributed to free volume variations in this homologous series.

\section{REFERENCES}

/1/Walker,W.W., Positron Annihilation, Edited by L. Dorikens-Van Praet, M. Dorikens and D. Segers, World Scientific Publishing Company, Singapore, 1989, p-860.

12/Lutton,E.S., Fatty Acids, 2nd Ed., Edited by K.S. Markley, Interscience, New York, 1960, Part 4, p-2583.

/3/Walker,W.W. and Harrell,J.W., Struct. Chem. 2 (1991) 359.

/4/Kirkegaard,P. and Eldrup,M., Comp. Phys. Commun. 7 (1974) 401.

15/Zerbi,G., Conti,G., Minoni,G., Pison,S., and Bigotto,A.,J. Phys. Chem. 91 (1987) 2386.

16/Feiser,L.F. and Feiser,M., Organic Chemistry, 3rd Ed., Reinhold Publishing Corp., New York, 1956, p-401. 